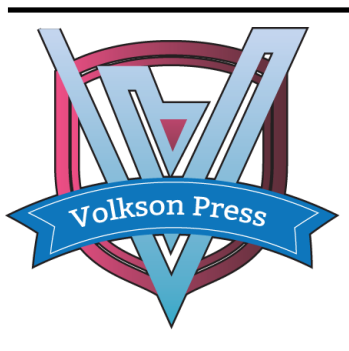

Contents List available at VOLKSON PRESS

New Materials and Intelligent Manufacturing (NMIM) DOI : http://doi.org/10.26480/icnmim.01.2018.136.138

Journal Homepage: https://topicsonchemeng.org.my/

ISBN: 978-1-948012-12-6

\title{
PREPARATION AND CHARACTERIZATION OF A NEW TYPE OF HIGH OXYGEN PERMEABLE SILOXANE HYDROGEL
}

\author{
Zhihui Zhu, Zhixun Lu, Huanhuan Zhang, Wei Dong* \\ Department of Materials Science and Engineering, Jilin Insitute of Chemical Technology Chengde Street 45, Jilin 132022, China. \\ *Corresponding Author Email:373308441@qq.com
}

This is an open access article distributed under the Creative Commons Attribution License, which permits unrestricted use, distribution, and reproduction in any medium, provided the original work is properly cited

\section{ARTICLE DETAILS}

Article History:

Received 26 June 2018

Accepted 2 July 2018

Available online 1 August 2018

\section{ABSTRACT}

In this study, a new type of siloxane hydrogel was prepared using $\alpha$-hydroxyethyl methacrylate (HEMA), $\mathrm{N}$ vinylpyrrolidone (NVP), and $\gamma$-Methacryloxypropyl trimethoxysilane (KH570) as main raw materials, and its properties have been characterized by Fourier transform infrared spectroscopy (FTIR), differential scanning calorimetry (DSC), and oxygen permeability. The investigated results illuminated that the hydrogel possessed good thermal stability and excellent oxygen permeability. According to these results, it could be found that the siloxane hydrogel had the significant potential to be used as contact lens material.

\section{KEYWORDS}

Siloxane hydrogel, Siloxane hydrogel, equilibrium moisture content

\section{INTRODUCTION}

Hydrogel was a hydrophilic three-dimensional network structure colloid that can retain a great quantity of water molecules or biological fluids [1]. Increasing studies have shown that hydrogel has similar characteristics to those of extracellular matrix and was easily regulated its physical or chemical properties by changing raw materials or synthetic methods to prepare a new type of hydrogel with better performance. For the above advantages, hydrogel has been widely used in biological area, especially in the field of contact lenses [2-3]. The design and development of the hydrogel material for contact lenses should ensure it possessed characteristics of well optical transparency, thermal stability and biocompatibility. What's more, for the cornea cannot get oxygen from the air duing to the lack of blood vessels, the materials should have high oxygen permeability to avoid oxygen supply poverty which causing corneal edema and leading to some eye diseases [4-6].

In study, we using NVP and KH570 as hydrophilic monomers to form oligomers, adding HEMA as major polymerized monomers and TEGDMA as coupling agent for bulk polymerization, designed and prepared a new type of high oxygen permeable siloxane hydrogel with hydrophilic interpenetrating network structure [7-10].

Our work provides a method for preparing siloxane hydrogel materials with good wettability, without plasma surface or other further chemical treatments, that may provide information valuable to the research and development of materials for contact lenses [11-12].

\section{EXPERIMENTAL SECTION}

\subsection{Materials and Instrumentations}

HEMA, KH570, NVP, AIBN and TEGDMA were purchased from Shanghai Titan technology Reagent Co., Ltd. (Shanghai, China). DSC measurements were performed on DSC-100 calorimeter (Mi Yuan Electric Co., Ltd., Shanghai, China). The image of sample was observed under a JSM-6300F scanning electron microscope (JEOL Co., Ltd., Japan).IR spectra was recorded on Nicolet6700 Fourier transform infrared (FTIR) spectrophotometer (Nicolet Co., Ltd., USA). Oxygen permeability test was performed on Y310 Oxygen Transmission Rate Tester (Biao Ji Packaging Equipment Co., Ltd., Guangzhou, China).

\subsection{Preparation of the siloxane hydrogelmaterial}

AIBN, NVP and KH570 were put into deionized water, then the solution was stirred at room temperature for $0.5 \mathrm{~h}$. Subsequently HEMA and TEGDMA were added to the solution above and was stirred for $1 \mathrm{~h}$ at room temperature. The reaction mixture solution was added to the polypropylene mould and reacting for 4 hours in loft drier at $100{ }^{\circ} \mathrm{C}$ after pressed film. Immersing the resulting product in deionized water for mold unloading after reaction completely and siloxane hydrogel material was obtained. Keep it soaking in the physiological saline (PH 7.4) for 2 hours before detection.

\section{RESULTS AND DISCUSSION}

\subsection{The characterization of the siloxane hydrogel complex}

The data of FTIR was shown in Figure 1. The spectrum of mixture before reaction exhibited a peak at about $3431.5 \mathrm{~cm}^{-1}$, corresponding to the telescopic vibration of the intramolecular hydroxyl group, the intense absorption peaks at $2955.7 \mathrm{~cm}^{-1}$ and $2875.8 \mathrm{~cm}^{-1}$ assigned to characteristic peak of $\mathrm{C}-\mathrm{H}$ stretching vibration (Fig. 1A) demonstrates the intense absorption, the intense absorption peaks at $1719.6 \mathrm{~cm}^{-1}$ and $1636.5 \mathrm{~cm}$ ${ }^{1}$ assigned to characteristic peaks of carbonyl group and carbon-carbon double bond intramolecular (Figure 1A). The new absorption peak at about $292.7 \mathrm{~cm}^{-1}$ corresponding to the change of $\mathrm{C}-\mathrm{H}$ stretching vibration indicated that the occurrence of chemical polymerization in the spectrum of mixture after reaction (Figure 1B). The disappearance of absorption peak at about $1620 \sim 1680 \mathrm{~cm}^{-1}$ corresponding to carbon-carbon double bond indicated that there were no monomers which not been reacted. 


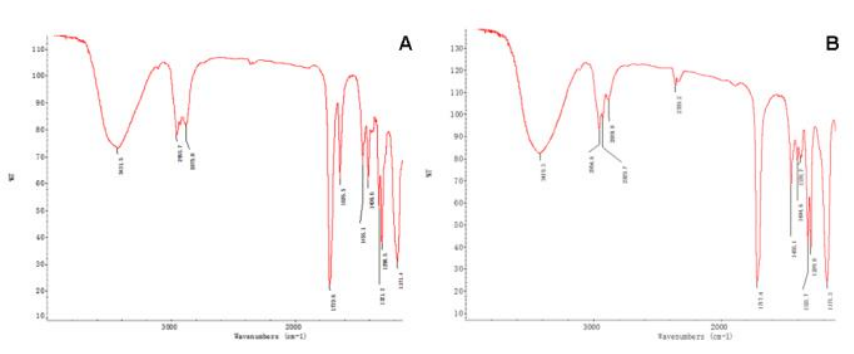

Figure 1: FTIR spectra of mixed solution of material before $(A)$ and after (B) reaction

The thermotropic behaviors hydrogel complexsprepared by different methods were recorded by DSC measurements (Figure 2).The DSC thermograms of materials prepared by monomers directly polymerizated diaslayed thermal absorption peak at $100^{\circ} \mathrm{C}$ suggested that the loss of water of hydration in the hydrogels as well as that of siloxane hydrogel complexs. Notably, the DSC thermogram of siloxane hydrogel complexs displayed dramatically different for the absence of thermal absorption peak at $250{ }^{\circ}$ Cindicated that the materials successfully covalent coupled. The DSC thermograms displayed thermopositive peak at $450{ }^{\circ} \mathrm{C}$ indicated that the hydrogel materials could not be resolved blow $450{ }^{\circ} \mathrm{C}$.

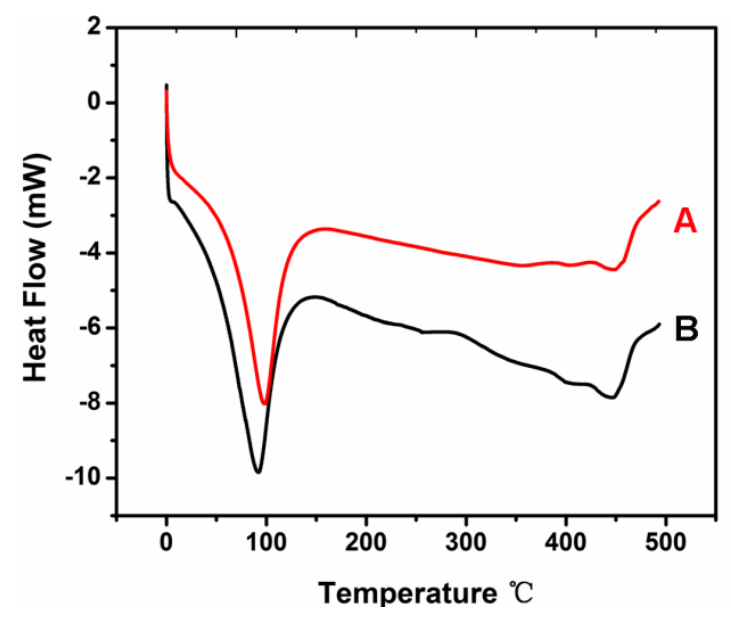

Figure 2: DSC thermograms of materials prepared by monomers directly and siloxane hydrogel complexes.

3.2 The effect of NVP content on the equilibrium moisture content of siloxane hydrogel contact lens materials

The positive correlation between the equilibrium moisture content of siloxane hydrogel and the content of NVP was shown in Figure 3. Although the monomers of NVP and HEMA were hydrophilic, the HEMA was easier to form hydrogen bonds with water molecules doing to plenty of hydroxyl groups on its branched chain, which induced the hydrodynamics volume of the polymer leading to the decreased of water absorption. The NVP with rigid structure destabilized the hydrogen bonds on the branched chains of HEMA causing the decreased of coupled. The swelling degree and structural stretch of hydrogel material increased with the NVP content, leading enough space for storing water molecules in the cross-linked structure, thus improving the equilibrium moisture content. As was shown in Figure 3, the percent of equilibrium moisture was greater than $40 \%$ as the percent of NVP over $10 \%$, which satisfied with the moisture content to be used.

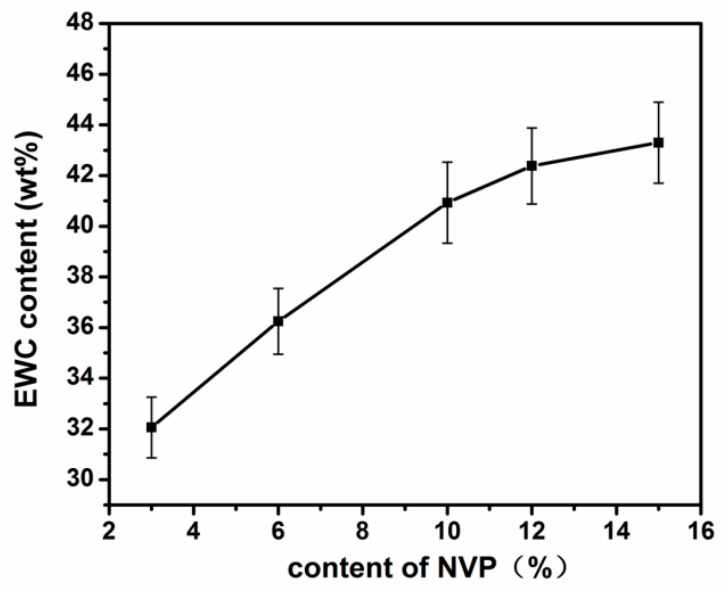

Figure 3: Relationship between the content of NVP and equilibrium moisture content of hydrogel materials

3.3 The effect of KH570 content on the equilibrium moisture content of siloxane contact lens materials

The equilibrium moisture content decreased with the increase of KH570 content (Figure 4), this may be due to the trimethylsiloxane hydrophobic group of KH570, while the decreasing of NVP and HEMA content, leading to the percent of hydrophilic functional groups reduced at the same time. What's more, as KH570 increased the intertwining nodes of polymer chains duing to its coupling effect, the space structure of the materials network became smaller and the mechanical properties decreased, resulting in the decreasing of the equilibrium swelling rate.As was shown in Figure 4, the percent of equilibrium moisture was greater than $40 \%$ as the percent of NVP less than $6 \%$, which satisfied with the moisture content to be used.

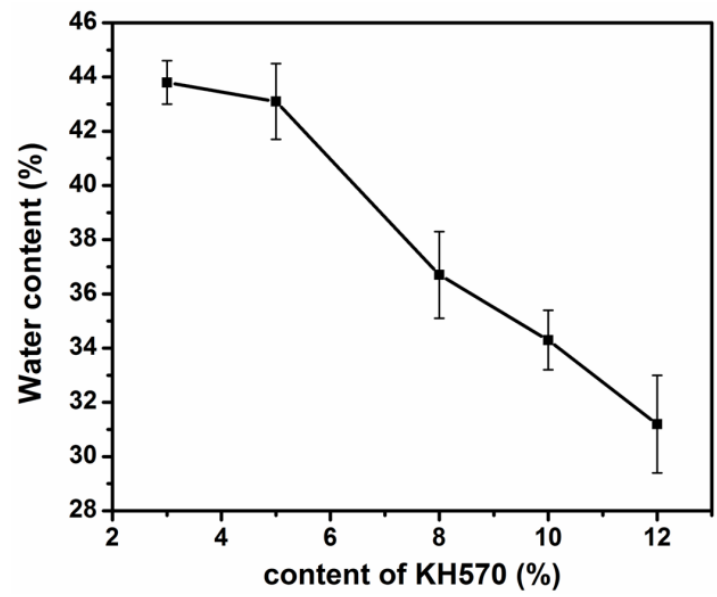

Figure 4: Relationship between the content of KH570 and equilibrium moisture content of hydrogel materials

3.4 The effect of molding temperature on the equilibrium moisture content of siloxane contact lens materials

The equilibrium moisture content increased with the increase of molding temperature was shown in Figure 5. Asa crosslinked polymer, the swelling process of hydrogel displayed the diffusion that of the solvent to crosslinked polymers. Water molecules could permeat into the material at lower temperature and the irregular heat movement of the water molecules was bound to the crystal lattice formed in the molding process. With the increase of the molding temperature, the crosslinking density of the siloxane hydrogel increased, which reduces the space for water molecules in the material, leading to the affinity between the hydrogel and the water molecules decreased. As was shown in Figure 5, the percent of equilibrium moisture was greater than $40 \%$ as the molding temperature over $110{ }^{\circ} \mathrm{C}$, which satisfied with the moisture content to be used. 


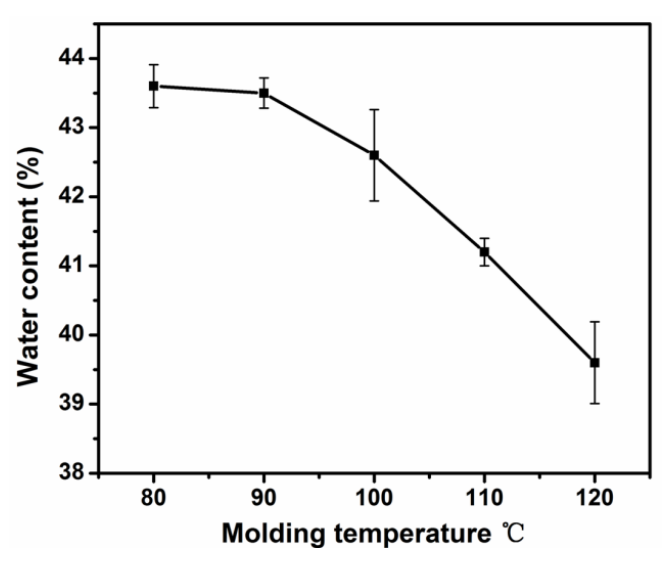

Figure 5: Relationship between the molding temperature and equilibrium moisture content of hydrogel materials

3.5 Water content and oxygen permeability of products formed by various ratios of monomers

According to the experimental results above, the hydrogel materials were prepared by the four monomers with various proportions, and the water content and oxygen permeable value have been measured. The data of the measurements were shown in Table 1 . It can be concluded that the synthesized hydrogel materials have good oxygen permeability and balanced water content, and the materials prepared by HEMA, NVP and KH570 monomers with the ratio of 7:2:1 displayed the highest water content and oxygen permeable value.

Table 1: Water content and oxygen permeability of materials

\begin{tabular}{|llc|}
\hline Ratios of monomers & $\begin{array}{l}\text { Water content } \\
\%\end{array}$ & $\begin{array}{l}\text { Oxygen } \\
\mathrm{DK} /\left(\mathrm{cm}^{2} / \mathrm{s} \times \mathrm{mLO}_{2} / \mathrm{mL}\right. \\
\mathrm{hPa}) \times 10^{-11}\end{array}$ \\
\hline HEMA: NVP:KH570=7:2:1 & 47.6 & 58.9 \\
HEMA: NVP:KH570=40:7:3 & 43.3 & 46 \\
HEMA: NVP:KH570=80:17:3 & 43.8 & 42 \\
HEMA: NVP:KH570=19:5:1 & 45.2 & 54.2 \\
\hline
\end{tabular}

\subsection{Image of various products recorded by SEM}

Several types of hydrogels were examined by SEM images (Figure 6). The cross section SEM images of the hydrogels synthesized in this study show that it has a directional comb structure, which conducive to the transport of water molecules and oxygen molecules (Figure 6A). The cross section of materials that prepared by monomers directly showed no directional structure which in favor of transporting water molecules and oxygen molecules (Figure 6B). We selected hydrogel corneal contact materials sold by well-known brands and processed SEM measurement on its cross sections. The images were shown in Figure 6C and Figure 6D.
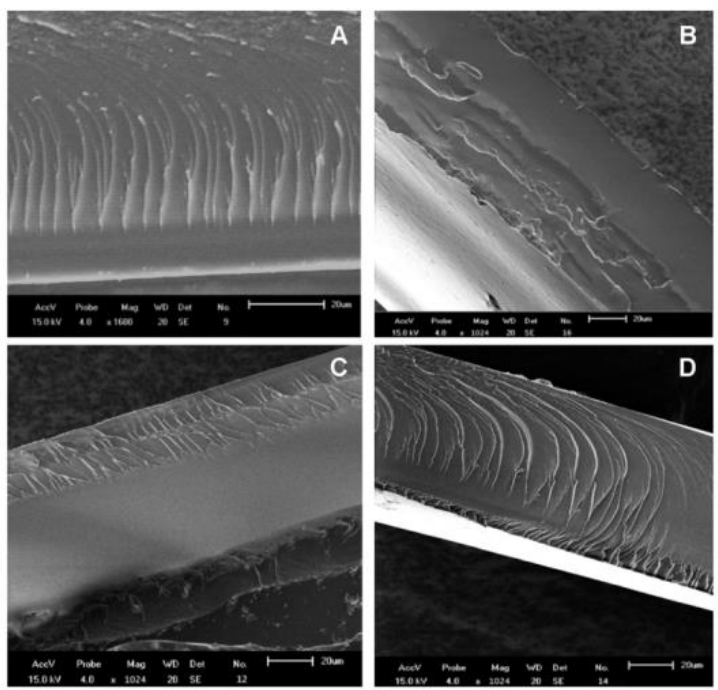

Figure 6: SEM images of siloxane hydrogel complexs (A), materials prepared by monomers directly (B) and hydrogel corneal contact materials sold by well-known brands (C, D).

\section{CONCLUSIONS}

In this study, we prepared a homogeneous network like hydrogel, it has good thermal stability and directionality in structure which is beneficial to the conduction of water molecules and oxygen molecules, and there is no monomores residual in the reaction process. All the properties of the materials are in accordance with the using standard the corneal contact lens materials. In the preparation process, we using series of measurements demonstrated that the water content of the materials was positively correlated with the content of NVP, while it has the reverse correlation with the content of KH570 and the molding temperature. The materials prepared by HEMA, NVP and KH570 monomers mixed with the ratio of 7:2:1 has the best properties of water retention and oxygen permeability.

\section{ACKNOWLEDGMENTS}

This work was financially supported by the Jilin Provincial Department of Science and Technology(20140204015GX).

\section{REFERENCES}

[1] McGill, M., Coburn, J.M., Partlow, B.P., Mu, X., Kaplan, D.L. 2017. Molecular and macro-scale analysis of enzyme-crosslinked silk hydrogels for rational biomaterial design. Acta biomaterialia, 63, 76-84.

[2] Kamata, H., Li, X., Chung, U.I., Sakai, T. 2015. Design of hydrogels for biomedical applications. Advanced healthcare materials, 4 (16), 23602374.

[3] Wang, Y. 2014. Synthesis and Properties of Silicone Hydrogel Contact Lens Materials. Applied Chemicals, 43 (2), 316-318.

[4] Dong, W., Zhu, Z., Chen, J., Jin, F., Kang, Z., Wang, J. 2017. Preparation method of fluorine-containing siloxane interpenetrating network hydrogel. Jilin, CN106950717A, 07-14.

[5] Rimmer, S. 2011. Synthesis of hydrogels for biomedical applications: control of structure and properties. In Biomedical Hydrogels, 51-62.

[6] Zhu, Z., Jiang, D., Yao, S., Dong, W. 2017. Preparation of fluorosilicone hydrogel materials and the influencing factors of water content. New chemical materials, 45 (9), 99-101.

[7] Chang, F., Vogt, J., Pruitt, J.D., Qian, X., Smith, D.A., Domschke, A.M., Wu, D. 2012. U.S. Patent No. 8,163,206. Washington, DC: U.S. Patent and Trademark Office.

[8] Qi, B. 2001. Contact Lenses Oxygen Permeation and Wearing Glasses for the Night. China Optical Technology Magazine, 1, 58-60.

[9] Zheng, R., Zhou, D. 2000. Contact Lens Research Progress. Chinese Journal of Practical Ophthalmology, 18 (5), 262-265.

[10] Guillon, M., Maissa, C. 2007. Use of silicone hydrogel material for daily wear. Contact Lens and Anterior Eye, 30 (1), 5-10.

[11] Zhang, X., Liu, M., Li, Y., Dong, Y., Pingguan-Murphy, B., Lu, T., Xu, F. 2015. Engineering cell microenvironment using novel functional hydrogels. European Polymer Journal, 72, 590-601.

[12] Pescosolido, L., Vermonden, T., Malda, J., Censi, R., Dhert, W. J., Alhaique, F., Matricardi, P. 2011. In situ forming IPN hydrogels of calcium alginate and dextran-HEMA for biomedical applications. Acta Biomaterialia, 7 (4), 1627-1633. 